\title{
Sports training enhances visuo-spatial cognition regardless of open-closed typology
}

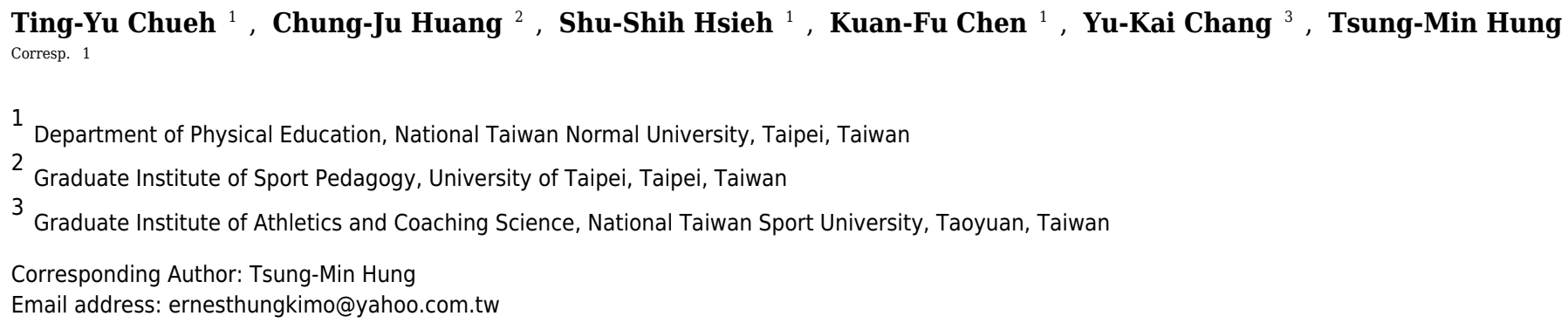

The aim of this study was to investigate the effects of open and closed sport participation on visuo-spatial attention and memory performance among young adults. Forty-eight young adults-16 open-skill athletes, 16 closed-skill athletes, and 16 non-athletes controlswere recruited for the study. Both behavioral performance and event-related potential (ERP) measurement were assessed when participants performed non-delayed and delayed match-to-sample task that tested visuo-spatial attention and memory processing. Results demonstrated that regardless of training typology, the athlete groups exhibited shorter reaction times in both the visuo-spatial attention and memory conditions than the control group with no existence of speed-accuracy trade-off. Similarly, a larger P3 amplitudes were observed in both athlete groups than in the control group for the visuo-spatial memory condition. These findings suggest that sports training, regardless of typology, are associated with superior visuo-spatial attention and memory performance, and more efficient neural resource allocation in memory processing. 


\section{Sports training enhances visuo-spatial cognition regardless of open-closed typology}

\section{Abstract}

4 The aim of this study was to investigate the effects of open and closed sport participation on

5 visuo-spatial attention and memory performance among young adults. Forty-eight young 6 adults-16 open-skill athletes, 16 closed-skill athletes, and 16 non-athletes controls-were recruited

7 for the study. Both behavioral performance and event-related potential (ERP) measurement were 8 assessed when participants performed non-delayed and delayed match-to-sample task that tested 9 visuo-spatial attention and memory processing. Results demonstrated that regardless of training 10 typology, the athlete groups exhibited shorter reaction times in both the visuo-spatial attention 11 and memory conditions than the control group with no existence of speed-accuracy trade-off. 12 Similarly, a larger P3 amplitudes were observed in both athlete groups than in the control group 13 for the visuo-spatial memory condition. These findings suggest that sports training, regardless of 14 typology, are associated with superior visuo-spatial attention and memory performance, and 15 more efficient neural resource allocation in memory processing. 


\section{Introduction}

A growing body of evidence shows that exercise training positively affects cognitive function (Hillman, Erickson, \& Kramer, 2008), particularly that which demands greater executive control. Athletes have superior sport performance and physical fitness due to prolonged engagement in sports training. According to the broad transfer hypothesis, extensive practice of specific skills can improve cognition for circumstances outside the specific sport context (Furley \& Memmert, 2011). One meta-analytical study has shown that athletes perform better on not only cognitive tasks with sport-related contexts relative to non-athletes, but also general cognitive tasks (Voss, Kramer, Basak, Prakash, \& Roberts, 2010). Studies have also

10 found that athletes outperform non-athletes in general executive control paradigms which tap on 11 motor inhibition and cognitive flexibility (Alves et al., 2013; Chan, Wong, Liu, Yu, \& Yan, 2011;

12 Taddei, Bultrini, Spinelli, \& Di Russo, 2012; Verburgh, Scherder, van Lange, \& Oosterlaan, 13 2014).

14 Schmidt and Wrisberg (2008) suggest that sports can be categorized into two types, 15 open-skill (e.g., racket sports, team sports) and closed-skill (e.g. jogging, swimming, cycling), 16 depending on the variability, predictability, and complexity of the performing environment. 17 Wang et al. (2013) found that open-skill sport athletes (tennis players) demonstrated better 18 response inhibition than closed-skill sport athletes (swimmers). However, Jacobson and 19 Matthaeus (2014) found that closed-skill sports athletes exhibited larger cognitive benefits in 20 interference control tasks than open-skill sports athletes, whereas open-skill sports athletes 21 outperformed closed-skill sports athletes in the problem-solving task. These results suggest that 22 the effect of sports types on cognitive benefit may depend on the specific cognitive domains.

23 Therefore, further examination of the relationships between sports types and different aspects of 24 executive control among athletes is warranted.

25 Visuo-spatial cognition is one aspect of higher-order cognition worth studying with respect 26 to sports training. Visuo-spatial cognition consists of a multi-faceted set of functions, such as 27 perception, selection, organization, and utilization of location and object-based information, and 28 offers a structure for how we interact with our physical environments (Possin, 2010). Athletes 29 are required to place high demands on visuo-spatial processing when they perform in the field 30 (Yarrow, Brown, \& Krakauer, 2009). Recent studies have found that sports training, particularly 
1 open-skill sports, might enhance cognition with higher visuo-spatial cognitive demand. For 2 example, Wang, Guo and Zhou (2016) found that table tennis athletes exhibited better 3 performance relative to non-athletes when they performed the attentional network test (ANT).

4 Additionally, Wang, Tsai, et al. (2015) found that badminton athletes not only exhibited shorter 5 reaction times in both visuo-spatial attention and memory conditions than non-athletes, but also 6 showed greater task-elicited modulations in beta power in the attention condition as well as in 7 theta and beta power in the memory condition. These results suggest that open-skill training 8 could facilitate visuo-spatial attention and memory performance at both behavioral and neuro-electrical levels.

10 Closed-skill sports with a high demand on physical fitness, cardiorespiratory fitness (CRF) 11 in particular (e.g. distance running, swimming, and triathlon), may also benefit visuo-spatial 12 cognition. Several studies with different research designs have shown the benefits of CRF 13 training (e.g., running, cycling) on visuo-spatial attention and memory. For example, one 14 intervention study has shown that six weeks of CRF training (i.e., running) enhanced 15 visuo-spatial memory among young adults (Stroth, Hille, Spitzer, \& Reinhardt, 2009). A cross-sectional study also showed that young adults with higher CRF demonstrated faster 17 response relative to those with lower CRF counterparts in the visuo-spatial attention task (i.e., 18 Posner paradigm) (Wang, Liang, et al., 2015). These results suggest benefits to visuo-spatial 19 attention and memory from both open-skill as well as closed-skill sport training with high 20 demand on CRF. However, none of the previous studies concurrently compared open- and 21 closed-skill training on visuo-spatial attention and memory function.

22 The employment of electrophysiological measures with high temporal resolution, such as 23 event-related potential (ERP), can provide further insight regarding the effects of sports training 24 on cognition. ERP offers a finer evaluation of distinct cognitive operations occurring between 25 stimulus encoding and response execution (Luck, Woodman, \& Vogel, 2000). The P3 (b) 26 component is the most frequently examined ERP component. It is the largest positive-going peak 27 waveform occurring approximately around $300 \mathrm{~ms}$ following stimulus onset, reflects cognitive 28 processing when attention and memory mechanisms are engaged, and relates to context-updating 29 (Johnson, 1993; Polich, 2007). The amplitude of P3 is associated with the amount of neural 30 resources being allocated to task-relevant stimuli, and latency is related to the timing of stimulus 
1 classification (Kutas, McCarthy, \& Donchin, 1977; Polich \& Kok, 1995). Previous ERP studies

2 in athletes focused mainly on response inhibition (i.e., Go-Nogo paradigm) and showed that 3 athletes not only exhibited shorter reaction times than non-athletes, but also larger amplitudes of

4 the N2 and P3 component in a No-go condition (Di Russo, Taddei, Apnile and Spinelli (2006).

5 Similar findings were found in middle-aged fencers (Taddei et al., 2012), and disabled athletes

6 (Di Russo et al., 2010). Altogether, these results suggest that open-skill sport training might

7 facilitate several neural correlates in motor inhibition.

8 However, to our knowledge, there have been no ERP studies that examine the effects of 9 sport training on visuo-spatial cognition, and the comparison between different sport types.

10 Therefore, the aim of this study was to investigate the effect of open and closed sport 11 participation on visuo-spatial attention and memory performance among young open-skill 12 athletes, closed-skill athletes, and non-athlete controls. This study employed the non-delayed and 13 delayed match-to-sample task with both behavioral and ERP measurements. In this task, 14 participants are required to engage visuo-spatial attention and memory processing that can 15 effectively elicit the P3 component (Müller \& Knight, 2002). We hypothesized that athlete 16 groups, regardless of sports typologies (i.e., open-skill and closed-skill), would exhibit shorter 17 reaction times than the control group in both visuo-spatial attention and memory condition, and 18 no existence of speed-accuracy trade off with similar results in accuracy-adjusted reaction times 19 and no accuracy difference among groups. Moreover, both athlete groups would show a larger 20 amplitude and shorter latency of P3 than the control group in both conditions. 


\section{Method}

\section{Participants}

Forty-eight participants were recruited from universities in Taipei. They were divided into

5 three groups based on their sport typology, and the three groups were matched in age and gender:

6 open-skill sports group (OS; $\mathrm{n}=16$, mean age $=20 \pm 1.2$ years), closed-skill sports group (CS; $\mathrm{n}$

$7=16$, mean age $=21.1 \pm 2.3$ years $)$, and non-athletes control group $(\mathrm{Con} ; \mathrm{n}=16$, mean age $=20.7$

$8 \pm 1.1$ years $)$. Participants in the OS group were badminton $(n=7)$ or table tennis $(n=9)$ athletes

9 while those in CS group were swimming $(n=7)$, triathlon $(n=1)$, and distance running $(n=8)$

10 athletes. The Con group was comprised of those without sport training experience. There was

11 one left-handed participant in each group based on Edinburgh handedness inventory scores

$12(\mathrm{OS}=66.8 \pm 46.7, \mathrm{CS}=77 \pm 43.4, \mathrm{Con}=60.5 \pm 45.4)$ (Oldfield, 1971). Athletes in both sport

13 groups were competing at national division 1 level and engaged in intensive training for at least

14 six months preceding their participation in the experiment. All participants met the following

15 criteria: They (a) were non-smokers; (b) had normal or corrected-to-normal vision; (c) did not

16 report diagnosed psychiatric or neurological disorders; (d) did not take medication that would

17 influence central nervous system functioning; and (e) were able to perform physical exercise

18 without discomfort or health risks based on an assessment with the Physical Activity Readiness

19 Questionnaire (PAR-Q). All participants were required to sign the written informed consent

20 approved by the Research Ethics Committee of National Taiwan Normal University

21 (201602HM005).

\section{Procedures}

23 Participants were instructed to visit the laboratory for two testing sessions. All sessions 24 were completed within one month and separated by at least one week. Participants were required 25 to refrain from food and drink consumption, except water, 1.5 hours before each session. In the 26 first session, participants first completed the demographic questionnaire, socio-economic status 27 of the family (SES) (Hollingshead \& Redlich, 1958), handedness inventory (Oldfield, 1971), 28 PAR-Q, International Physical Activity Questionnaire (IPAQ) (Liou, Jwo, Yao, Chiang, \& 29 Huang, 2008), and informed consent form. Then participants were instructed to sit on a 30 comfortable chair and fitted with an electrode cap in a quiet and dimly lit data acquisition room. 
1 Afterwards, participants were provided cognitive task instructions and performed practice trials.

2 The formal data recording commenced when participants reached an accuracy rate of $80 \%$ in the 3 practice trials. In the second session, participants were administered a non-verbal IQ test using

4 Raven's Progressive Matrices: SPM Plus Sets (Styles, Raven, \& Raven, 1998). Next, participants'

5 height and weight were measured, and the cardiorespiratory fitness measurement was

6 administered. Participants were given US \$30 compensation after they completed the second

7 session.

8 Measures

Cardiorespiratory Fitness Assessment. Cardiorespiratory fitness by peak oxygen consumption (VO2 peak) was measured for each participant utilizing the Bruce Treadmill

11 Protocol, which is a maximal graded exercise test (GXT) on a motorized treadmill. During this

12 protocol, both the speed and slope increased every 3 min until participants were exhausted, and

13 the test was terminated when at least two of following three criteria were met: (a) a plateau in

14 VO2 with increasing exercise intensity; (b) a respiratory exchange ratio above 1.10; and (c)

15 HRmax within 15 beats of age-predicted HRmax (220-age) (American College of Sports 16 Medicine, 2006; Howley, Bassett, \& Welch, 1995).

17 Cognitive Assessments. This study employed a modified non-delayed and delayed 18 match-to-sample test adapted from Wang and Tsai (2016) to examine visuo-spatial attention 19 (non-delayed condition) and visuo-spatial memory (delayed condition) function (see Figure1).

20 The task was programmed with STIM 2.0 software (Neuroscan Ltd, El Paso, TX, USA). All 21 stimuli were presented on a 17-inch computer monitor that was placed $60 \mathrm{~cm}$ in front of 22 participants. The stimuli consisted of a red dot $\left(0.5^{\circ} \times 0.5^{\circ}\right)$ randomly presented within a $3.8^{\circ} \times$ $237.4^{\circ}$ gray rectangle. One dot could appear in any one of nine locations (i.e., center, center right, 24 center left, upper center, upper right corner, upper left corner, lower center, lower right corner, 25 and lower left corner) within its rectangle. Participants were instructed to determine whether the 26 location of the red dots appeared in the same position within their respective rectangles.

27 In the visuo-spatial attention condition (non-delayed), two rectangles were presented 28 simultaneously; one rectangle was placed in the center of the screen, while the other was placed 29 either to the left or to the right of the center. The two rectangles were presented for $180 \mathrm{~ms}$, a 30 duration shorter than is typical for voluntary saccades, to minimize the potential effects of 
1 unwanted saccades on the results (Müller \& Knight, 2002). In the visuo-spatial memory

2 condition (delayed), the stimulus 1 (S1) was presented for $180 \mathrm{~ms}$ with an equal probability on 3 either the left or right of the central fixation $\left(0.5^{\circ} \times 0.5^{\circ}\right)$, followed by a 3 -second delay. Stimulus

42 (S2) then appeared for a duration of $500 \mathrm{~ms}$ in the center of the screen. Participants were

5 instructed to retain the position of the S1 red dot in their memory during the 3-second delay and

6 then determine whether its position was identical to the position of the red dot in S2.

7 The response time windows were $2000 \mathrm{~ms}$ for both conditions. Participants pressed the 8 "YES" button with their left thumb when the position of red dots within each gray rectangle were 9 identical and they pressed the "NO" button with their right thumb when they were not. 10 Participants were provided feedback on each response ('correct', 'incorrect') immediately after 11 the $2000 \mathrm{~ms}$ response period. Before the formal test, participants were reminded that accuracy 12 and speed were equally important, and instructed to achieve $80 \%$ of response accuracy on the 13 practice trials. A total of 240 trials were equally divided into four blocks, and were randomly 14 presented non-delayed and delayed conditions with equal probability. Rest intervals between 15 blocks were between three to five minutes.

16 The behavior data were analyzed to derive the response accuracy, median reaction times 17 (RT), and the intra-individual variability in RT, for evaluation of behavior performances. The 18 median RT was measured on correct trials and intra-individual variability in RT using the 19 intra-individual coefficient of variation formula (ICV= the SD of RT / the mean of RT). We used 20 median RT to alleviate potential artifacts deriving from higher rates of outliers that 21 disproportionally contribute to mean RT. In addition, RT distributions are usually positively

22 skewed. Median RT is less sensitive to the skew of distribution (Baayen \& Milin, 2010). The 23 response accuracy was calculated as the ratio between number of correct responses and total 24 number of trials. In addition, the accuracy-adjusted RT was computed using the median $25 \mathrm{RT} /$ accuracy rate formula to avoid the potential influence of a speed-accuracy trade-off strategy 26 on task performance (Sutherland \& Crewther, 2010).

27 Electroencephalographic Recording. Electroencephalographic (EEG) activity was 28 recorded with 30 electrode sites using an elastic electrode cap (Quick-Cap, Compumedics 29 Neuroscan, Inc., Charlotte, NC, USA). The electrode sites were mounted according to the 30 modified International 10-10 System (Chatrian, Lettich, \& Nelson, 1985). The 
1 electrooculographic (EOG) activity was measured by using 4 electrodes placed at the outer 2 canthus of each eye, and above and below the left orbit. An average of the mastoid (A1, A2) 3 served as the reference, and FPz was set as the ground electrode on the Quick-Cap. All electrode 4 impedances were below $5 \mathrm{k} \Omega$. The EEG data acquisition was performed with a sampling rate of $51000 \mathrm{~Hz}$, using a DC- to $200-\mathrm{Hz}$ filter and a 60-Hz notch filter.

6 For data reduction, the EOG activity was corrected using the algorithm described by 7 (Semlitsch, Anderer, Schuster, \& Presslich, 1986). Epochs were defined as 100 ms pre- stimulus 8 to $1000 \mathrm{~ms}$ post-stimulus, and baseline corrections were performed using the 100-ms 9 pre-stimulus interval. The data were filtered using a $30-\mathrm{Hz}$ low-pass cutoff ( $12 \mathrm{~dB} /$ octave), and 10 with an amplitude outside the range of $\pm 100 \mu \mathrm{V}$ were excluded at any electrode. After visual 11 inspection, only trials with correct responses were averaged. The P3 amplitude was measured 12 defined as the first maximal positive peak around or after 300ms following stimulus onset in 13 non-delayed condition and stimulus 2 onset in delayed condition and was measured from the 14 midline electrode sites (i.e., Fz, $\mathrm{Cz}, \mathrm{Pz}$ ) for each participant, and latency was detected as the time 15 point corresponding to the maximum peak amplitude.

\section{Statistical Analysis}

18 Data analyses were performed using the SPSS 21.0 software system. One-way ANOVAs were separately computed to test homogeneity of the demographic variables height, weight, non-verbal IQ, handedness scores, socio-economic status (SES) of the family, video game experience, physical activity level, and cardiorespiratory fitness among groups. The independent t-test was conducted to compare the training experience and daily training hours for the past six months between two athlete groups. The two way Group (OS, CS, and Con) x Condition

24 (Non-delay and Delayed) repeated-measures ANOVAs were separately performed on behavioral 25 data (i.e., median RT, ICV, response accuracy, and accuracy-adjusted RT) to examine group 26 differences in behavioral performance. The three way Group (OS, CS, and Con) x Condition 27 (Non-delay and Delayed) x Site $(\mathrm{Fz}, \mathrm{Cz}$, and $\mathrm{Pz}$ ) repeated-measures ANOVAs were performed 28 on the P3 amplitude and latency to examine group differences in neuro-electrical performance. 29 Post-hoc comparisons were conducted using LSD significant difference tests. An alpha $=.05$ was 30 set as the level of statistical significance for all analyses. Eta-squared effect sizes $\left(\eta^{2}\right)$ were 
1 reported for significant main effects and interactions, and a Greenhouse-Geisser correction was 2 used to adjust for violations of the sphericity assumption.

3 


\section{Results}

\section{Demographic data}

3 Table 1 presents the participants' characteristics. No significant differences were observed

4 in height $(F(2,45)=0.158, p=.854)$, weight $(F(2,45)=0.522, p=.597)$, handedness scores

$5 \quad(F(2,45)=0.536, p=.589)$, hours playing video games per week in past years $(F(2,45)=2.256$,

$6 p=.144)$, and socio-economic status of the family $(F(2,45)=2.415, p=.101)$ among groups. There

7 was a significant difference in non-verbal IQ $\left(F(2,45)=11.70, p<.05, \eta^{2}=.342\right)$, and a post hoc

8 comparison revealed that the control group had a higher non-verbal IQ than both athlete groups,

9 but no significant difference between the two athlete groups was observed.

10 With regards to sport characteristics, there was no difference between the two athlete groups

11 in terms of the number of years engaged in sport training $(t(30)=1.094, p=.283)$. The

12 closed-skill group had longer daily training hours within the past six months than the open-skill

13 group $\left(t(16.659)=-2.64, p<.05, \eta^{2}=0.188\right)$. Both athlete groups had greater physical activity

14 levels than the control group $\left(F(2,45)=47.142, p<.05, \eta^{2}=.677\right)$, but no significant difference

15 existed between the two athlete groups. Furthermore, there were significant differences in

16 cardiorespiratory fitness across the three groups $\left(F(2,45)=11.10, p<.05, \eta^{2}=.33\right)$, and a post hoc

17 comparison demonstrated that the closed-skill group had the highest cardiorespiratory fitness,

18 followed by the open-skill group and the control group, but there was only a marginal difference

19 between the open-skill and control group $(F(1,30)=4.865, p=.06)$. 


\section{Behavioral data}

Figure 2 presents the results for response accuracy, RT, ICV, and accuracy-adjusted RT.

3 The response accuracy revealed a Condition effect $\left(F(1,45)=93.398, p<.05, \eta^{2}=.447\right)$, with a 4 higher accuracy in the non-delayed condition (95.91\%) than in the delayed condition (89.05\%).

5 There were no significant effects of Condition by Group $(F(2,45)=.310, p=.735)$, and Group $6(F(2,45)=0.025, p=.096)$ for accuracy results. Furthermore, the RT results revealed the main 7 effects of Condition $\left(F(1,45)=9.61, p<.05, \eta^{2}=.166\right)$ and $\operatorname{Group}\left(F(2,45)=5.11, p<.05, \eta^{2}=.185\right)$. 8 RT in the delayed condition $(658.76 \mathrm{~ms})$ was shorter than in the non-delayed condition (690.71 $\mathrm{ms}$ ) and both athlete groups exhibited shorter RT than the control group regardless of the 10 condition, but there was no difference between two athlete groups (OS: $632.52 \mathrm{~ms} \&$ CS: 655.03 $11 \mathrm{~ms}<$ Con: $736.66 \mathrm{~ms})$. There were no significant effects of Condition by Group $(F(2,45)=1.576$, $12 p=.218)$ for RT results. Regarding ICV, only a significant effect in Condition $(F(1,45)=50.379, p$ $\left.13<.05, \eta^{2}=.511\right)$ was observed, and the delayed condition (0.242) had a higher ICV than the 14 non-delayed condition (0.198). There were no significant effects of Condition by Group $15(F(2,45)=1.499, p=.234)$, and Group $(F(2,45)=0.113, p=.894)$ for ICV results. Similar results 16 were found for RT and accuracy-adjusted RT, with a significant effect of Group $(F(2,45)=4.988$, $17 p<.05, \eta^{2}=.181$ ). Both athlete groups had a shorter accuracy-adjusted RT than the control group 18 regardless of condition, but there was no difference between the two athlete groups (OS: 6.85 $19 \mathrm{~ms} / \%$ \& CS: $7.11 \mathrm{~ms} \%$ < Con: 8 ms $\%$ ). There were no significant effects of Condition by Group $20 \quad(F(2,45)=.817, \quad p=.448$, and Condition $(F(1,45)=1.92, p=.173)$.

21 An additional analysis was performed on RT that included cardiorespiratory fitness and 22 daily training hours as a covariate to compare the two athlete groups by using two-way Group 23 (OS and CS) X Condition (Non-delay and Delayed) repeated-measures ANCOVAs. The result 24 also showed no significant difference between the two athlete groups $(F(1,28)=1.485, p=.233)$ 25 (OS: $622.05 \mathrm{~ms} \& \mathrm{CS}: 665.5 \mathrm{~ms})$.

\section{ERP data}

28 Figure 3 illustrates the grand average ERP results at $\mathrm{Fz}, \mathrm{Cz}$, and $\mathrm{Pz}$ for each group and each condition. For the P3 amplitude, there was a significant main effect of Electrode $(F(1.419$, $\left.63.875)=121.113, p<.05, \eta^{2}=.725\right)$, and an interaction of Condition and Group $(F(2,45)=3.453, p$ 
$\left.1<.05, \eta^{2}=0.129\right)$. A post hoc comparison revealed that $\mathrm{Pz}(11.67 \mu \mathrm{V})$ had the largest amplitude,

2 followed by $\mathrm{Cz}(7.52 \mu \mathrm{V})$ and $\mathrm{Fz}(3.512 \mu \mathrm{V})$. Further decomposition of the Group by Condition

3 interaction revealed a significant Condition effect. Both athlete groups had a larger amplitude

4 than the control group in the delayed condition $\left(F(2,45)=4.520, p<.05, \eta^{2}=.167\right)(\mathrm{OS}: 8.157 \mu \mathrm{V}$

$5 \& \mathrm{CS}: 9.301 \mu \mathrm{V}>\mathrm{Con}: 5.244 \mu \mathrm{V})$, but there was no significant difference in the non-delayed

6 condition $(F(2,45)=0.864, p=.428)$. There was no significant effect of Condition $(F(1,45)=0.065$,

$7 p=.801)$ or interactions of Group and Electrode $(F(4,90)=0.502, p=.734)$, Electrode and

8 Condition $(F(1.61,72.441)=3.070, p=.063)$, or Group, Electrode and Condition $(F(4,90)=1.014$,

$9 p=.405)$.

10 Regarding the P3 latency, there were no significant effects of Electrode $11(F(1.653,74.371)=0.802, \quad p=.431) \quad$ or $\quad$ Condition $\quad(F(1,45)=0.394, \quad p=.534) \quad$ or Group $12(F(2,45)=0.033, p=.968)$ or interactions of Group and Electrode $(F(4,90)=1.283, p=.282)$, 13 Condition and Electrode $(F(1.542,69.398)=2.061, p=.146)$, Group and Condition $14(F(2,45)=0.018, p=.983)$, or Group, Condition and Electrode $(F(4,90)=1.016, p=.404)$.

15 An additional analysis was performed on the P3 amplitude that included cardiorespiratory 16 fitness and daily training hours as a covariate in the delayed condition to compare the two athlete 17 groups by using two-way Group (OS and CS) X Sites (Fz, Cz, and Pz) repeated-measures 18 ANCOVAs. The result also showed no significant difference between the two athlete groups $19(F(1,28)=0.009, p=.926)(\mathrm{OS}: 8.81 \mu \mathrm{V} \quad \& \quad \mathrm{CS}: 8.65 \mu \mathrm{V})$.

\section{Relationship between RT and the P3 amplitude averaged across midline sites}

The correlation analysis was performed to examine whether RT and accuracy-adjusted RT were correlated with the P3 amplitude averaged across midline sites (i.e., Fz, Cz and Pz) in each condition among all participants. In the delayed condition, the P3 amplitude were significantly correlated with RT $(r=-.349, p<.05)$ and accuracy-adjusted RT $(r=-.313, p<.05)$, but not in the non-delay condition for RT ( $r=-.112, p=.447)$ and accuracy-adjusted RT $(r=-.126, p=.392)$. 


\section{Discussion}

The aim of this study was to investigate the effects of open and closed sport participation on visuospatial attention and memory performance using behavioral and neuro-electrical measures among young open-skill athletes, closed-skill athletes, and non-athletes controls. The main findings were that regardless of their sport typology, athletes exhibited shorter reaction times than the non-athletes in both the visuo-spatial attention and memory conditions, and no existence of speed-accuracy trade off with similar results in accuracy-adjusted reaction times and no accuracy difference among groups. Furthermore, both athlete groups demonstrated a larger P3 amplitude in the visuo-spatial memory condition relative to the control group. Our findings suggest that both open- and closed-skill sport training are associated with superior visuo-spatial attention and memory performance, and better neural resource allocation during memory 12 neurocognitive processing.

13 Both athlete groups outperformed the non-athletes group on tasks that required visuo-spatial 14 attention and memory processes, which advances existing knowledge. The current study was 15 consistent with previous findings showing that athletes exhibit superiority not only in sports-related contexts (Mann, Williams, Ward, \& Janelle, 2007) but also in general cognitive

17 functions (Alves et al., 2013; Jacobson \& Matthaeus, 2014; Taddei et al., 2012; Verburgh et al., 18 2014; Wang et al., 2013). Given that previous studies in athlete and cognition have focused on 19 the executive function domains with less visuo-spatial demands, the present study adds to the 20 literature by employing a cognitive task that requires more engagement of visuo-spatial 21 processing (Wang, Tsai, et al., 2015). Furthermore, the employment of a cognitive task with 22 conditions that varied in processing complexity verified that athletes committed to prolonged 23 open- and close-skilled sport training are associated with superior visuo-spatial attention and memory processing at both the perceptual and imperative levels.

25 The finding of a larger P3 amplitude in both athlete groups, compared to the non-athletes 26 group, suggest that athletes invested greater neural resources for the evaluation/classification of 27 imperative stimuli during the retrieval phase of visuo-spatial memory condition than the controls. 28 These results are consistent with previous work focusing on motor inhibition which found that 29 open-skill athletes (i.e., fencers) exhibited a larger P3 component during a No-go condition (Di 30 Russo et al., 2006; Taddei et al., 2012). Within the present study, the P3 amplitude was 
1 significantly correlated with RT and accuracy-adjusted RT in the visuo-spatial memory condition,

2 but not in the visuo-spatial attention condition. These findings suggest that sports training might 3 facilitate visuo-spatial memory performances, at least in part, by the modulation of neural 4 resource allocation to task-relevant stimuli. Moreover, the finding of no difference in P3 5 amplitude between the open- and closed-skill groups implies that prolonged sport training, irrespective of training modality, is associated with enhanced neural resources allocation during

7 the visuo-spatial memory processes.

8 Notably, the absence of a training effect on the P3 amplitude during the visuo-spatial attention condition is worthy of further exploration. Previous studies have indicated that fencers

10 demonstrated a larger P3 amplitude in No-go condition relative to non-athlete controls, whereas 11 no group difference in P3 amplitude was observed for Go stimuli during a Go/no go paradigm

12 (Di Russo et al., 2006; Taddei et al., 2012). This indicates that the cognitive benefits of training 13 effects might be observed with higher mental loads. In terms of visuo-spatial processing, a 14 greater investment of cognitive resources is required for memory than for attention (Gazzaley \& 15 Nobre, 2012). These results, in concert with our findings, indicate that sport training-elicited 16 benefits for neural resource allocation may be specific to cognitive domains with higher mental 17 loads (i.e., visuo-spatial memory).

18 With respect to P3 latency, the current study found no significant group differences. This 19 result was consistent with that of a previous study which utilized a Go/No-go paradigm (Taddei 20 et al., 2012) and found no significant difference in the P3 latency between athletes and controls, 21 whereas a group difference was revealed in the P3 amplitude. In addition, Wang and Tsai (2016) 22 found that individuals with higher levels of physical activity exhibited a larger P3 amplitude than 23 those with lower levels during visuo-spatial processing, but no group difference was found in the 24 P3 latency. Accordingly, we speculate that sport training enhances visuo-spatial cognitive 25 performances, particularly those with higher mental demand, through the modulation of neural 26 resource allocation, not the speed of stimulus evaluation/classification.

27 Unlike previous studies (Wang \& Tsai, 2016; Wang, Tsai, et al., 2015) that demonstrated 28 how the memory (delayed) condition had a higher accuracy and a longer RT than the attention 29 (non-delayed) condition across groups in a non-delayed and delayed match-to-sample task, the 30 current study showed that the RT was shorter in the memory condition than the attention 
1 condition, which is inconsistent with the results of past studies. We also revealed that the 2 memory condition exhibited higher intra-individual variability in RT (ICV) than the attention 3 condition. As far as the authors are concerned, these results could be interpreted in relation to the 4 higher unpredictability and complexity of the imperative stimulus during the memory paradigm, 5 which resulted in higher uncertainty and impulsive responses, as indexed by greater 6 intra-individual ICV and shorter RT.

7 There are several limitations of this study. First, its cross-sectional design prevents causal 8 inferences. Second, the current study applied non-delayed and delayed match-to-sample tasks 9 that included visual-spatial attention and memory processing, which omitted the examination of 10 other high-order cognitions. Third, we could not exclude all confounding factors that could bias 11 the relationship between sport type and cognition despite the control of several confounding 12 variables, such as CRF and daily training hours, among two athlete groups. The current study 13 recruited athletes that were mostly from the department of physical education, in which case they 14 may have participated in different types of exercise in addition to formal training. Previous 15 studies have shown that participation in exercise is beneficial to cognitive function (Guiney \& 16 Machado, 2013). Accordingly, it is possible that recreational exercise participation played a 17 critical role in biasing the association of sport typology with cognitive function in athletes, which 18 should be carefully considered in future related studies. Fourth, the response sides were not 19 counterbalanced. Although this issue is unlikely to affect our observed effect because all the 20 behavioral measures were pooled across the response side in the present study, future research 21 should consider counterbalancing the response side. Finally, we recruited collegiate athletes, 22 which may limit the generalizability of findings to athletes from different ages (e.g., 23 adolescents). 


\section{Conclusions}

3 In conclusion, the current study demonstrated that regardless of sport typology, athletes 4 exhibited superior visuo-spatial attention and memory performance relative to non-athletes at the 5 behavioral level. Furthermore, the training-elicited benefits can be extended to neuro-electrical 6 level of visuo-spatial memory processing. Our findings not only provide convergent evidence for 7 the broad transfer hypothesis that athletes' expertise can be transferred from sports-specific 8 contexts to general cognitive contexts, but also shed light on the association of sport type with 9 superior visuo-spatial attention and memory performances and greater allocation of neural 10 resource during memory processing. 


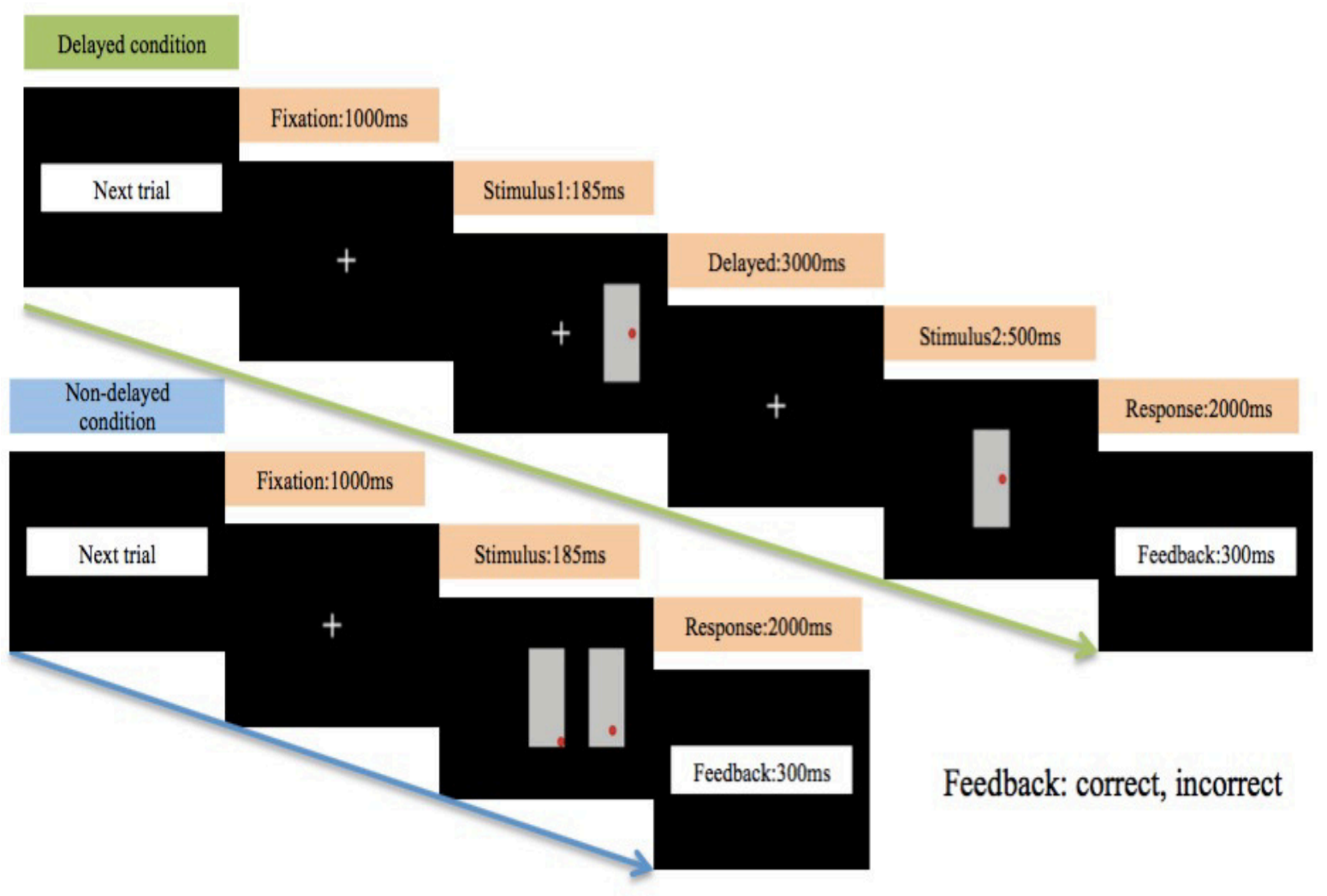

4 Figure 1. Illustration of the non-delay and delayed match-to-sample task

5

6 


\begin{tabular}{|c|c|c|c|c|}
\hline Variables & Open-skill $(\mathrm{n}=16)$ & Closed-skill (n=16) & Control $(n=16)$ & Total $(\mathrm{n}=48)$ \\
\hline Female & 7 & 7 & 7 & $21 / 48$ \\
\hline Left-handed & 1 & 1 & 1 & $3 / 48$ \\
\hline Age (years) & $20(1.2)$ & $21.1(2.3)$ & $20.7(1.1)$ & $20.6(1.6)$ \\
\hline Height $(\mathrm{cm})$ & $170.2(9.4)$ & $170.7(6.7)$ & $169.0(9.1)$ & $170.0(8.3)$ \\
\hline Weight (kg) & $63.9(11.8)$ & $61.5(10.1)$ & $59.9(11.7)$ & $61.8(11.1)$ \\
\hline Non-verbal IQ test & $38.3(4.2)^{*}$ & $41.7(5.1)$ & $46.7(5.4)$ & $42.2(5.9)$ \\
\hline Socio-economic status of family & $2.1(0.8)$ & $2.5(0.9)$ & $1.9(0.7)$ & $2.2(0.8)$ \\
\hline $\begin{array}{l}\text { Video game experience in recently } \\
\text { six months } \\
\text { (week/hours) }\end{array}$ & $6.3(3.3)$ & $6.9(3.5)$ & $10.9(4.7)$ & $8.5(4.4)$ \\
\hline Training years & $10.8(2.2)$ & $9.7(3.2)$ & 0 & $10.2(2.8)$ \\
\hline $\begin{array}{l}\text { Daily training hours in recently six } \\
\text { months }\end{array}$ & $8.7(1.3)^{*}$ & $12.3(5.3)$ & 0 & $10.2(2.8)$ \\
\hline Total Physical activity level (MET) & $9078.6(2257.1)^{*}$ & $9154.0(3642.9)$ & $1702.2(1234.2)$ & $6645.2(1234.2)$ \\
\hline Cardiorespiratory fitness (m1/kg/min) & $46.2(7.2)^{*}$ & $55.8(11.9)$ & $39.6(9.7)$ & $47.2(11.7)$ \\
\hline
\end{tabular}

2 Note. 1 The number in parentheses is the standard deviation. ; $2 .{ }^{*}=$ group effect $; 3$. Metabolic equivalent $=$ MET 3 
(A)

Reaction times

- Delay -Non-delay

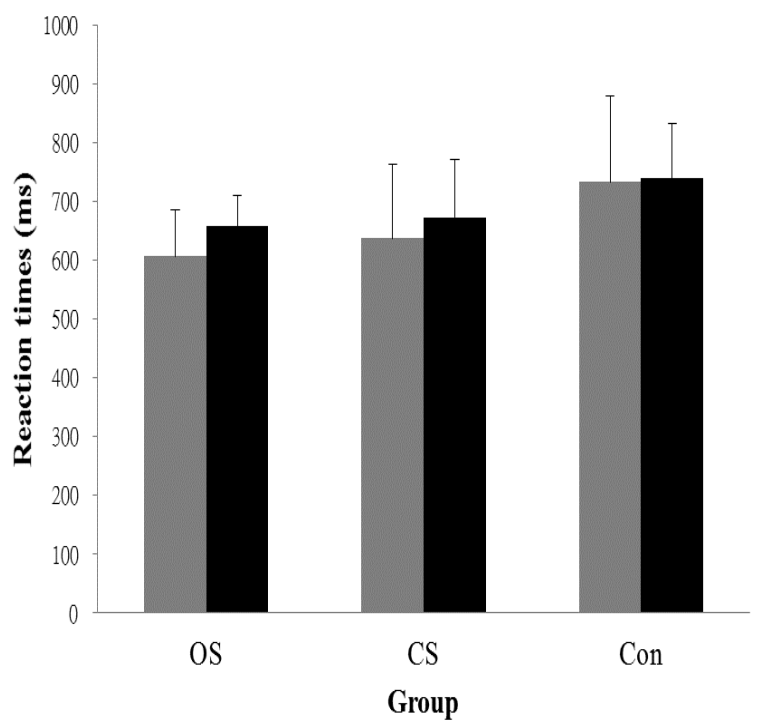

(C)

Accuracy-adjusted RT

- Delay Non-delay

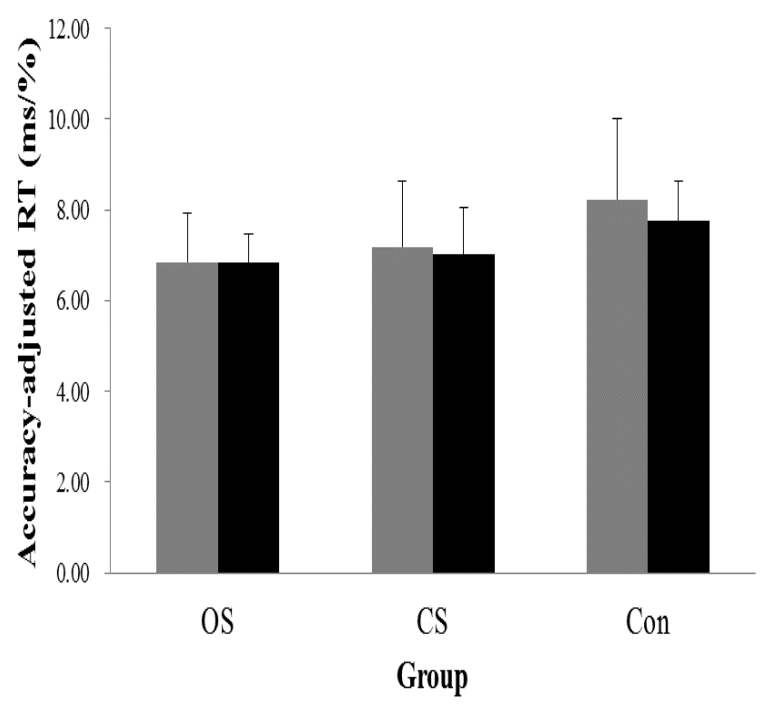

(B) Accuracy rate

-Delay - Non-delay

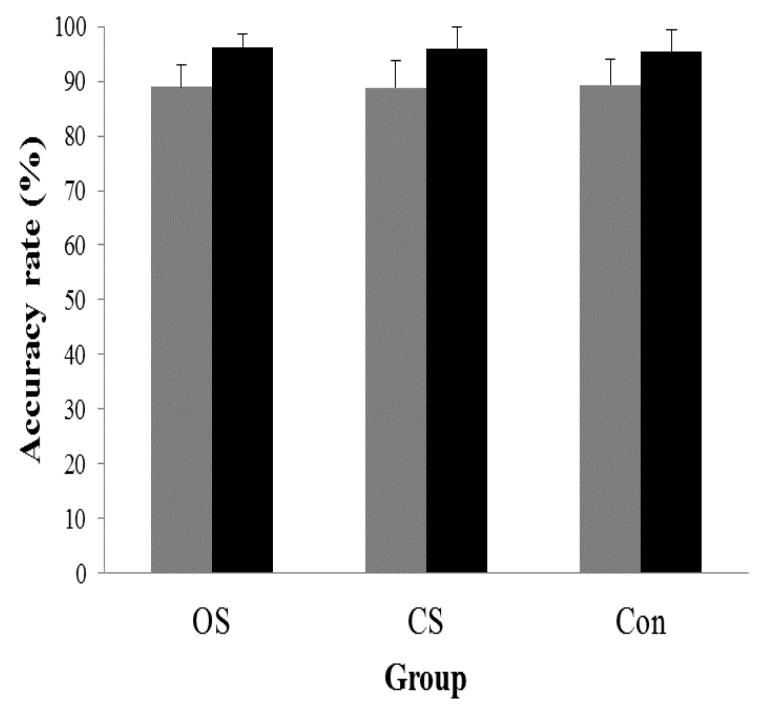

(D)
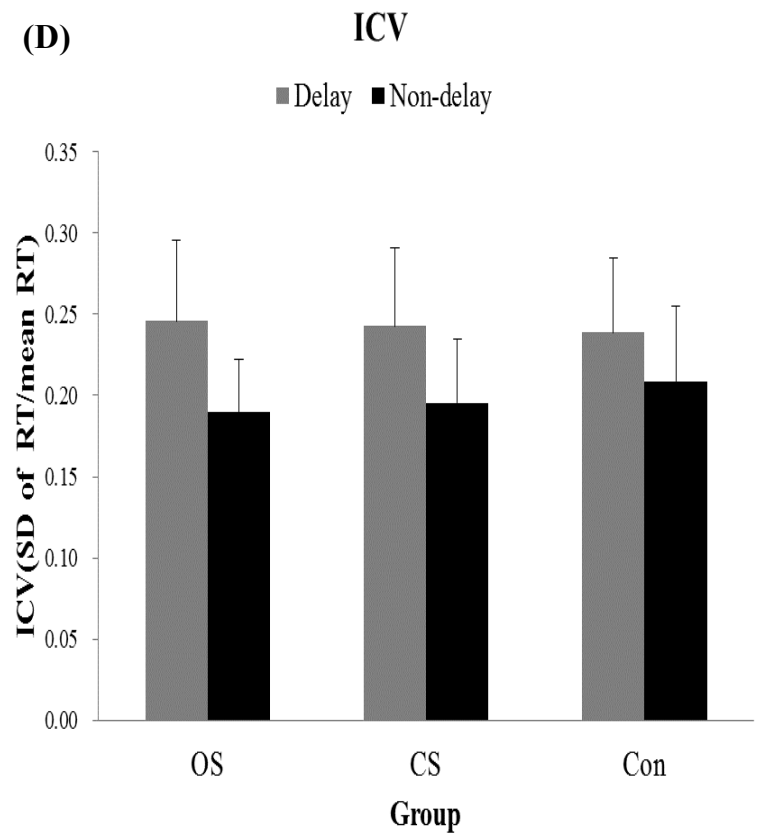

2 Figure 2 Behavioral data for each group (Mean \pm SD), A) Main effect of group and condition 3 of the RT, B) Main effect of condition of the Accuracy Rate, C) Main effect of group of the 4 Adjusted RT, D) Main effect of condition of the ICV in RT. 
1

\section{Delay-condition}

(A)

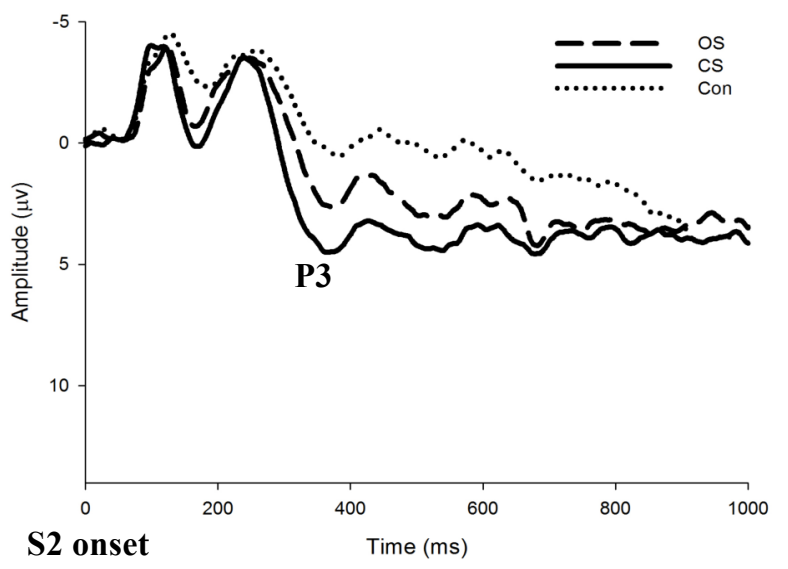

(B)

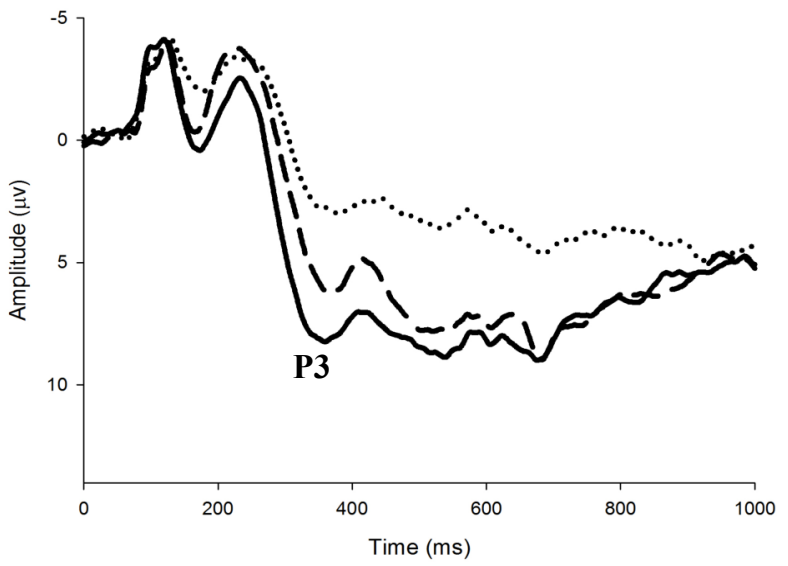

(C)

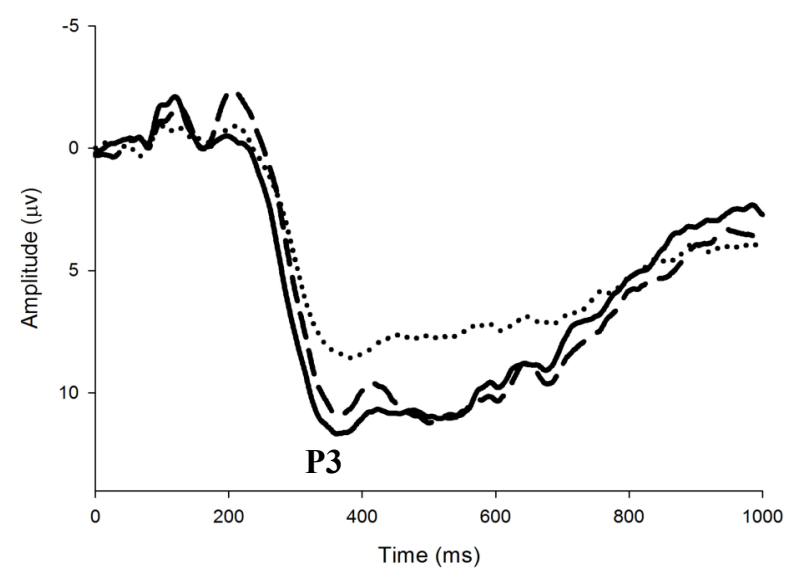

Non-delay condition

(D)

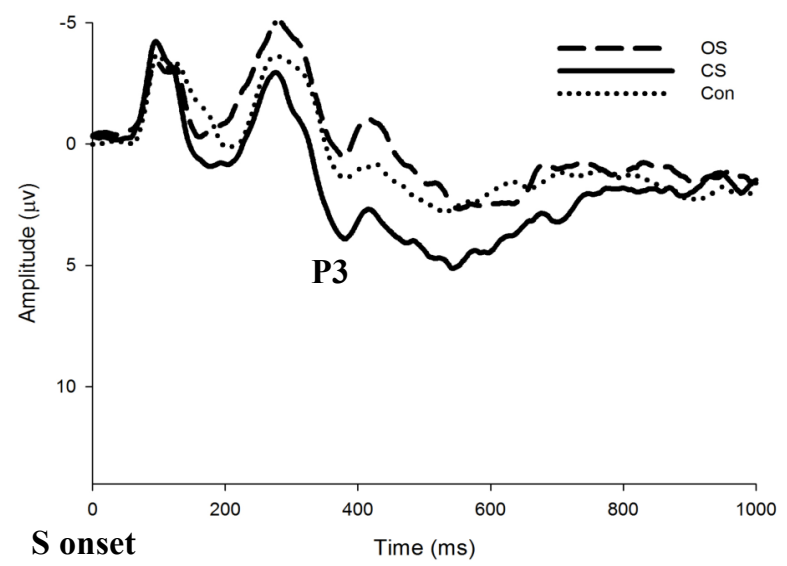

(E)

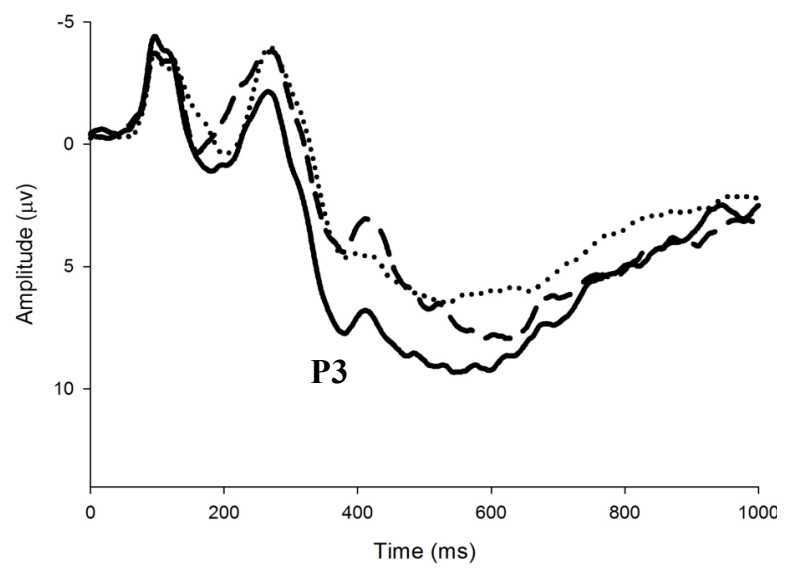

(F)

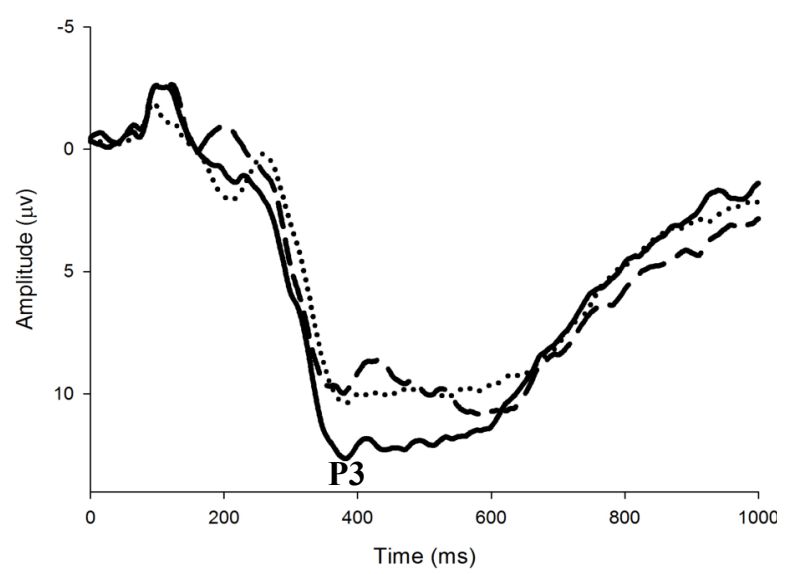

Figure 3 Grand average ERP at Fz, Cz, and Pz sites stratified by group for Delay condition (A, B, C) and Non-condition (D, E, F). 


\section{Reference}

2 Alves, H., Voss, M. W., Boot, W. R., Deslandes, A., Cossich, V., Salles, J. I., \& Kramer, A. F. (2013). Perceptual-cognitive expertise in elite volleyball players. Frontiers in psychology, 4(36), 1-9. DOI: 10.3389/fpsyg.2013.00036

American College of Sports Medicine. (2006). ACSM's guidelines for exercise testing and prescription. (7th ed.). Philadelphia, PA: Lippincott Williams \& Wilkins.

Baayen, R. H., \& Milin, P. (2010). Analyzing reaction times. International Journal of Psychological Research, 3(2), 12-28. DOI: 10.21500/20112084.807

Chan, J. S., Wong, A. C., Liu, Y., Yu, J., \& Yan, J. H. (2011). Fencing expertise and physical fitness enhance action inhibition. Psychology of Sport and Exercise, 12(5), 509-514. DOI: 10.1016/j.psychsport.2011.04.006

Chatrian, G., Lettich, E., \& Nelson, P. (1985). Ten percent electrode system for topographic studies of spontaneous and evoked EEG activities. American Journal of EEG technology, 25(2), 83-92.

Di Russo, F., Bultrini, A., Brunelli, S., Delussu, A. S., Polidori, L., Taddei, F.... (2010). Benefits of sports participation for executive function in disabled athletes. J Neurotrauma, 27(12), 2309-2319. DOI: 10.1089/neu.2010.1501

Di Russo, F., Taddei, F., Apnile, T., \& Spinelli, D. (2006). Neural correlates of fast stimulus discrimination and response selection in top-level fencers. Neurosci Letters, 408(2), 113-118. DOI: 10.1016/j.neulet.2006.08.085

Furley, P., \& Memmert, D. (2011). Studying cognitive adaptations in the field of sport: Broad or narrow transfer? . Perceptual and Motor skills, 113(2), 481-488. DOI: 10.2466/05.23.PMS.113.5.481-488

Gazzaley, A., \& Nobre, A. C. (2012). Top-down modulation: bridging selective attention and working memory. Trends in Cognitive Sciences, 16(2), 129-135. DOI: 10.1016/j.tics.2011.11.014

Guiney, H., \& Machado, L. (2013). Benefits of regular aerobic exercise for executive functioning in healthy populations. Psychonomic Bulletin and Review, 20(1), 73-86. DOI: 10.3758/s13423-012-0345-4

Hillman, C. H., Erickson, K. I., \& Kramer, A. F. (2008). Be smart, exercise your heart: exercise effects on brain and cognition. Nature Reviews Neuroscience, 9(1), 58-65. DOI: 
10.1038/nrn2298

Hollingshead, A. B., \& Redlich, F. C. (1958). Social class and mental illness: Community study. New York: Wiley.

Howley, E. T., Bassett, D. R., \& Welch, H. G. (1995). Criteria for maximal oxygen uptake: review and commentary. Medicine and Science in Sports and Exercise, 27(9), 1292-1301.

Jacobson, J., \& Matthaeus, L. (2014). Athletics and executive functioning: How athletic participation and sport type correlate with cognitive performance. Psychology of Sport and Exercise, 15(5), 521-527. DOI: 10.1016/j.psychsport.2014.05.005

Johnson, R. (1993). On the neural generators of the P300 component of the event-related potential. Psychophysiology, 30, 90-97.

Kutas, M., McCarthy, G., \& Donchin, E. (1977). Augmenting mental chronometry: the P300 as a measure of stimulus evaluation time. Science, 197(4305), 792-795.

Liou, Y. M., Jwo, C. J., Yao, K. G., Chiang, L.-C., \& Huang, L.-H. (2008). Selection of appropriate Chinese terms to represent intensity and types of physical activity terms for use in the Taiwan version of IPAQ. Journal of Nursing Research, 16(4), 252-263. DOI: 10.1097/01.JNR.0000387313.20386.0a

Luck, S. J., Woodman, G. F., \& Vogel, E. K. (2000). Event-related potential studies of attention. Trends in Cognitive Sciences, 4(11), 432-440. DOI: 10.1016/S1364-6613(00)01545-X

Mann, D. T., Williams, A. M., Ward, P., \& Janelle, C. M. (2007). Perceptual-cognitive expertise in sport: a meta-analysis. Journal of Sport \& Exercise Psychology, 29(4), 457-478. DOI: 10.1123/jsep.29.4.457

Müller, N. G., \& Knight, R. T. (2002). Age-related changes in fronto-parietal networks during spatial memory: An ERP study. Cognitive Brain Research, 13(2), 221-234. DOI: 10.1016/S0926-6410(01)00119-7

Oldfield, R. C. (1971). The assessment and analysis of handedness: The Edinburgh inventory. Neuropsychologia, 9(1), 97-113.

Polich, J. (2007). Updating P300: an integrative theory of P3a and P3b. Clinical Neurophysiology, 118(10), 2128-2148. DOI: 10.1016/j.clinph.2007.04.019

Polich, J., \& Kok, A. (1995). Cognitive and biological determinants of P300: An integrative review. Biological Psychology, 41(2), 103-146. DOI: 10.1016/0301-0511(95)05130-9 
1 Possin, K. L. (2010). Visual spatial cognition in neurodegenerative disease. Neurocase, 16(6), 466-487. DOI: $10.1080 / 13554791003730600$

3 Schmidt, R. A., \& Wrisberg, C. A. (2008). Motor learning and performance: A situation-based learning approach. Champaign, IL: Human Kinetics.

Semlitsch, H. V., Anderer, P., Schuster, P., \& Presslich, O. (1986). A solution for reliable and valid reduction of ocular artifacts, applied to the P300 ERP. Psychophysiology, 23(6), 695-703.

Stroth, S., Hille, K., Spitzer, M., \& Reinhardt, R. (2009). Aerobic endurance exercise benefits memory and affect in young adults. Neuropsychol Rehabil, 19(2), 223-243. DOI: $10.1080 / 09602010802091183$

Styles, I., Raven, J. C., \& Raven, M. (1998). Raven's Progressive Matrices: SPM Plus Sets AE. San Antonio: Harcourt Assessment.

Sutherland, A., \& Crewther, D. P. (2010). Magnocellular visual evoked potential delay with high autism spectrum quotient yields a neural mechanism for altered perception. Brain A Journal of Neurology, 133(7), 2089-2097. DOI: 10.1093/brain/awq122

Taddei, F., Bultrini, A., Spinelli, D., \& Di Russo, F. (2012). Neural correlates of attentional and executive processing in middle-age fencers. Medicine and Science in Sports and Exercise, 44(6), 1057-1066. DOI: 10.1249/MSS.0b013e31824529c2

Verburgh, L., Scherder, E. J., van Lange, P., \& Oosterlaan, J. (2014). Executive functioning in highly talented soccer players. PLoS One, 9(3), e91254. DOI: 10.1371/journal.pone.0091254

Voss, M. W., Kramer, A. F., Basak, C., Prakash, R. S., \& Roberts, B. (2010). Are expert athletes 'expert' in the cognitive laboratory? A meta-analytic review of cognition and sport expertise. Applied Cognitive Psychology, 24(6), 812-826. DOI: 10.1002/acp.1588

Wang, B., Guo, W., \& Zhou, C. (2016). Selective enhancement of attentional networks in college table tennis athletes: a preliminary investigation. PeerJ, 4, e2762. DOI: $10.7717 /$ peerj. 2762

Wang, C.-H., Chang, C.-C., Liang, Y.-M., Shih, C.-M., Chiu, W.-S., Tseng, P.... (2013). Open vs. closed skill sports and the modulation of inhibitory control. PLoS One, 8(2), e55773. DOI: 10.1371/journal.pone.0055773 
1 Wang, C.-H., Liang, W.-K., Tseng, P., Muggleton, N. G., Juan, C.-H., \& Tsai, C.-L. (2015). The relationship between aerobic fitness and neural oscillations during visuo-spatial attention

(1)

4

Wang, C.-H., \& Tsai, C.-L. (2016). Physical activity is associated with greater visuospatial

6

(1)

Sport \& Exercise Psychology, 38(1), 69-81. DOI: 10.1123/jsep.2015-0221

3
C.-H., Tsai, C.-L., Tu, K.-C., Muggleton, N. G., Juan, C.-H., \& Liang, W.-K. (2015).

Modulation of brain oscillations during fundamental visuo-spatial processing: A

comparison between female collegiate badminton players and sedentary controls.
Yarrow, K., Brown, P., \& Krakauer, J. W. (2009). Inside the brain of an elite athlete: the neural Psychology of Sport and Exercise, 16, 121-129. DOI: 10.1016/j.psychsport.2014.10.003 processes that support high achievement in sports. Nature Reviews Neuroscience, 10(8),

